\title{
Real Parametrization of Algebraic Curves *
}

\author{
J. Rafael Sendra ${ }^{1}$ and Franz Winkler ${ }^{2}$ \\ 1 Dpto de Matemáticas, Universidad de Alcalá de Henares, E-28871 Madrid, Spain \\ mtsendra@alcala.es \\ 2 RISC-Linz, J. Kepler Universität Linz, A-4040 Linz, Austria \\ Franz.Winkler@risc.uni-linz.ac.at
}

\begin{abstract}
There are various algorithms known for deciding the parametrizability (rationality) of a plane algebraic curve, and if the curve is rational, actually computing a parametrization. Optimality criteria such as low degrees in the parametrization or low degree field extensions are met by some parametrization algorithms. In this paper we investigate real curves. Given a parametrizable plane curve over the complex numbers, we decide whether it is in fact real. Furthermore, we discuss methods for actually computing a real parametrization for a parametrizable real curve.
\end{abstract}

\section{Introduction}

In [?], [?] we have described a symbolic algorithm for computing a rational parametrization $(x(t), y(t))$ of a plane algebraic curve $\mathcal{C}$ of genus 0 (only these curves have a rational parametrization). This algorithm is implemented in the system CASA [?]. Approaches to the parametrization problem for algebraic curves are also described in [?] and [?].

Definition: Let $\mathbb{K}$ be a field of characteristic $0, \mathcal{K}$ the algebraic closure of $\mathbb{K}$. Let the irreducible affine curve $\mathcal{C}$ be defined as the set of solutions in the affine plane $\mathbb{A}(\mathcal{K})^{2}$ of the polynomial equation

$$
f(x, y)=0
$$

over $\mathbb{K}$, i.e. $f \in \mathbb{K}[x, y]$. Then $x(t), y(t)$ in $\mathcal{K}(t)$, the field of rational functions over $\mathcal{K}$, constitute a rational parametrization of $\mathcal{C}$, if and only if, except for finitely many exceptions, every evaluation $\left(x\left(t_{0}\right), y\left(t_{0}\right)\right)$ at $t_{0} \in \mathcal{K}$ is a point on $\mathcal{C}$, and, conversely, almost every point on $\mathcal{C}$ is the result of evaluating the parametrization at some element of $\mathcal{K}$.

In this case $\mathcal{C}$ is called parametrizable or rational.

Equivalently, $\mathcal{P}(t)=(x(t), y(t))$ is a rational parametrization of $\mathcal{C}$ if $\mathcal{P}$ : $\mathbb{K} \longrightarrow \mathcal{C}$ is rational and not both $x(t)$ and $y(t)$ are constant. Furthermore, if $\mathcal{P}$ is birational we say that $\mathcal{P}(t)$ is a proper parametrization.

\footnotetext{
* The first author was supported by DGICYT PB 95/0563 and UAH-Proj. E010/97. The second author was supported by the Austrian Fonds zur Förderung der wissenschaftlichen Forschung under Proj. HySaX, P11160-TEC. Both authors were supported by the Austrian-Spanish exchange program Acción Integrada 30/97.
} 
The parametrization problem for algebraic curves consists in first deciding whether the given curve $\mathcal{C}$ has such a rational parametrization, and if so finding one.

In our geometrical approach to parametrization we basically determine the singularities of the curve $\mathcal{C}$, decide the genus of $\mathcal{C}(\mathcal{C}$ can be parametrized if and only if $\operatorname{genus}(\mathcal{C})=0$ ), find a couple of simple points on $\mathcal{C}$ in a low degree (i.e. degree 1 or 2 ) algebraic extension $\mathbb{K}(\gamma)$ of $\mathbb{K}$, and from the singularities and these simple points derive a parametrization of $\mathcal{C}$. The parametrization will have coefficients in $\mathbb{K}(\gamma)$. So starting with a parametrizable curve defined by a polynomial $f(x, y) \in \mathbb{Z}[x, y]$ we will either get a parametrization with rational coefficients or with coefficients in $\mathbb{Q}(\gamma), \gamma$ algebraic over $\mathbb{Q}$ of degree 2 . If the curve $\mathcal{C}$ is a real curve, i.e. if $\mathcal{C}$ has infinity many real points, then for obvious reasons we will not be satisfied with a parametrization with complex coefficients.

For practical applications, such as in computer aided geometric design, we need to be able to parametrize real curves with real coefficients, if possible. In this paper we demonstrate that if $\mathcal{C}$ is a real curve, then our algorithm actually computes a parametrization of $\mathcal{C}$ with real coefficients. Alternatively, one could take a possibly complex parametrization and, if possible, transform it to a real one. This approach is developed in [?] and [?].

\section{Real curves}

Let $f(x, y) \in \mathbb{C}[x, y]$ be a non-constant polynomial. $f$ defines a plane affine curve $\mathcal{C}$ over the complex numbers, i.e. $\mathcal{C} \subset \mathbb{A}^{2}(\mathbb{C})$, the affine plane over $\mathbb{C}$. Whenever useful or necessary, we will also consider the curve $\mathcal{C}$ in the projective plane, i.e. $\mathbb{P}^{2}(\mathbb{C})$. Points in the projective plane are written as $(a: b: c)$.

Definition: The curve $\mathcal{C}$ is a called a real curve, if and only if $\mathcal{C}$ has infinitely many points in $\mathbb{A}^{2}(\mathbb{R})$.

A real curve always has a defining polynomial over the reals. A proof of the following Lemma is given in [?].

Lemma 1: If the curve $\mathcal{C}$ is real, then it can be defined by a real polynomial.

Not only can every real curve be defined over the reals, also the irreducibility of the curve is independent from whether we view it in $\mathbb{A}^{2}(\mathbb{R})$ or in $\mathbb{A}^{2}(\mathbb{C})$. A proof of the following Lemma is given in [Wi96], Theorem 5.5.3.

Lemma 2: Let $\mathcal{C}$ be a real curve. $\mathcal{C}$ is irreducible over $\mathbb{R}$ if and only if it is irreducible over $\mathbb{C}$.

The algorithm presented in [?] implies that every parametrizable plane curve over an algebraically closed field $\mathcal{K}$ of characteristic zero can be parametrized over any subfield of $\mathcal{K}$ that contains the coefficients of the irreducible polynomial defining the curve, and the coordinates of one simple point of the curve. Thus, as a consequence of Lemma 1, one deduces that every real parametrizable plane curve can be parametrized over the reals (this result is also known as the algebraic version of the real Lüroth's theorem [?]), and that a parametrizable 
plane curve is real if and only if it has at least one real simple point.

Example 1: Let the curve $\mathcal{C}_{1}$ ([Wi96]) be defined by

$$
f_{1}(x, y)=\left(x^{2}+4 y+y^{2}\right)^{2}-16\left(x^{2}+y^{2}\right)=0 .
$$

$\mathcal{C}_{1}$ is a real curve. In fact $\mathcal{C}_{1}$ is a parametrizable real curve, and a parametrization over the reals is

$$
x(t)=\frac{-1024 t^{3}}{256 t^{4}+32 t^{2}+1}, \quad y(t)=\frac{-2048 t^{4}+128 t^{2}}{256 t^{4}+32 t^{2}+1} .
$$

On the other hand, the curve $\mathcal{C}_{2}$ ([?]) defined by

$$
f_{2}(x, y)=2 y^{2}+x^{2}+2 x^{2} y^{2}=0
$$

is not a real curve. The only point of $\mathcal{C}_{2}$ in the affine plane over the reals is the double point $(0,0)$. The complex curve $\mathcal{C}_{2}$ is parametrizable, and a parametrization is

$$
x(t)=\frac{-t^{2}-2 t+1}{t^{2}-2 t-1}, \quad y(t)=\frac{i t^{2}+2 i t-i}{2 t^{2}+2} .
$$

\section{A real parametrization algorithm}

Let $\mathbb{L}$ be a computable subfield of $\mathbb{C}$, and let the irreducible affine curve $\mathcal{C}$ be defined by the polynomial $f(x, y) \in \mathbb{L}[x, y]$. We may assume that $f$ is a real polynomial (indeed associated with a real polynomial), for otherwise by Lemma 1 one knows that $\mathcal{C}$ is not a real curve. Thus, we may suppose that $\mathbb{L}$ is a subfield of $\mathbb{R}$. In the process of parametrization it is necessary to view $\mathcal{C}$ as a projective curve in the projective plane $\mathbb{P}^{2}(\mathbb{C})$. This projective curve, also denoted by $\mathcal{C}$, is defined by the homogeneous polynomial $F(x, y, z) \in \mathbb{L}[x, y, z]$, where $F$ is the homogenization of $f$.

The property of parametrizability is independent of whether we view $\mathcal{C}$ in the affine or the projective plane, and parametrizations can be easily converted [?].

Definition: Let the irreducible curve $\mathcal{C}$ of degree $d$ be defined by the irreducible polynomial $f(x, y) \in \mathbb{L}[x, y]$ of degree $d$. The singular point $P \in \mathbb{P}^{2}(\mathbb{C})$ of multiplicity $m$ on $\mathcal{C}$ is an ordinary singular point, if and only if there are $m$ different tangents to $\mathcal{C}$ at $P$.

If $\mathcal{C}$ has only ordinary singularities $P_{1}, \ldots, P_{n}$ of multiplicities $r_{1}, \ldots, r_{n}$, respectively, then the genus of $\mathcal{C}$ is defined as

$$
\operatorname{genus}(\mathcal{C})=\frac{1}{2}\left[(d-1)(d-2)-\sum_{i=1}^{n} r_{i}\left(r_{i}-1\right)\right] .
$$

This definition, and also the method described in this paper, can be extended to curves with non-ordinary singularities. For the sake of simplicity we do not consider this situation here. 
The linear system of adjoint curves of degree $d^{\prime}$ to $\mathcal{C}$ consists of all the curves of degree $d^{\prime}$ having every point $P_{i}, 1 \leq i \leq n$, as a point of multiplicity at least $r_{i}-1$.

Since the adjoint curves of any degree $d^{\prime}$ to a rational plane curve $\mathcal{C}$ have defining polynomial over $\mathbb{L}$, and can be computed in a finite number of ground field operations [?], the problem of parametrizing is reduced to the problem of determining a simple point on the curve. We will do that by transforming $\mathcal{C}$ birationally to a conic $\mathcal{D}$. The simple real points on $\mathcal{C}$ and on $\mathcal{D}$ correspond uniquely to each other, except for finitely many exceptions. So there is a simple real point on $\mathcal{C}$ if and only if there is a simple real point on $\mathcal{D}$. This question can be decided. If the answer is yes, a real point on $\mathcal{D}$ can be computed, transformed to a real point on $\mathcal{C}$, and from this point we can derive a parametrization of $\mathcal{C}$ over $\mathbb{R}$.

In [?] we prove the following generalization of a theorem by Hilbert and Hurwitz [?].

Theorem 1: Let $\mathcal{C}$ be a rational plane curve of degree $d$ defined by a polynomial over $\mathbb{L}, \mathcal{H}_{a}$ the linear system of adjoint curves to $\mathcal{C}$ of degree $a \in\{d, d-1, d-2\}$, and $\tilde{\mathcal{H}}_{a}^{s}$ a linear subsystem of $\mathcal{H}_{a}$ of dimension $s$ with all its base points on $\mathcal{C}$. Then we have the following:

(i) If $\Phi_{1}, \Phi_{2}, \Phi_{3} \in \tilde{\mathcal{H}}_{a}^{s}$ are such that the common intersections of the three curves $\Phi_{i}$ and $\mathcal{C}$ are the set of base points of $\tilde{\mathcal{H}}_{a}^{s}$, and such that

$$
\mathcal{T}=\left\{y_{1}: y_{2}: y_{3}=\Phi_{1}: \Phi_{2}: \Phi_{3}\right\}
$$

is a birational transformation, then the birationally equivalent curve to $\mathcal{C}$, obtained by $\mathcal{T}$, is irreducible of degree $s$.

(ii) Those values of the parameters for which the rational transformation $\mathcal{T}$ is not birational satisfy some algebraic conditions.

We will use Theorem 1 to transform the curve $\mathcal{C}$ to either a line or a conic. For the transformed curves it will be easy to decide the existence of real points and if so to determine a real point. So we need to select a linear subsystem of low dimension in the system of adjoint curves, e.g. by fixing additional base points. These additional base points will introduce algebraic coefficients into the system, unless we can find rational ones or whole conjugate families of such points.

Definition: Let $F \in \mathbb{L}\left[x_{1}, x_{2}, x_{3}\right]$ be a homogeneous polynomial defining a parametrizable projective curve $\mathcal{C}$. Let $p_{1}, p_{2}, p_{3}, m \in \mathbb{L}[t]$. The set of projective points $\mathcal{F}=\left\{\left(p_{1}(\alpha): p_{2}(\alpha): p_{3}(\alpha)\right) \mid m(\alpha)=0\right\} \subset \mathbb{P}^{2}(\mathbb{C})$ is a family of $s$ conjugate simple points on $\mathcal{C}$ if and only if the following conditions are satisfied: $m$ is squarefree, $\operatorname{deg}(m)=s, \operatorname{deg}\left(p_{i}\right)<\operatorname{deg}(m)$ for $i=1,2,3, \operatorname{gcd}\left(p_{1}, p_{2}, p_{3}\right)=1$, $\mathcal{F}$ contains exactly $s$ points of $\mathbb{P}^{2}(\mathbb{C}), F\left(p_{1}(t), p_{2}(t), p_{3}(t)\right)=0 \bmod m(t)$, and there exists $i \in\{1,2,3\}$ such that $\frac{\partial F}{\partial x_{i}}\left(p_{1}(t), p_{2}(t), p_{3}(t)\right) \bmod m(t) \neq 0$. 
If we choose all the points in a family of conjugate points as additional base points in the system of adjoint curves, then the corresponding subsystem will again have coefficients over the ground field $\mathbb{L}$.

Definition: Let $\mathcal{C}$ be a plane curve defined by a polynomial over $\mathbb{L}, \mathcal{H}$ a linear system of curves in which all the elements are of the same degree, $\tilde{H}$ the defining polynomial of a linear subsystem $\tilde{\mathcal{H}}$ of $\mathcal{H}$, and let $\tilde{\mathcal{S}}$ be the set of base points of $\tilde{\mathcal{H}}$ that are not base points of $\mathcal{H}$. Then, we say that $\tilde{\mathcal{H}}$ is a rational subsystem of $\mathcal{H}$ if the following conditions are satified:

(1) $\tilde{H}$ has coefficients in $\mathbb{L}$.

(2) For almost every curve $\Phi \in \mathcal{H}$, and $\tilde{\Phi} \in \tilde{\mathcal{H}}$ it holds that

$$
\operatorname{dim}(\mathcal{H})-\operatorname{dim}(\tilde{\mathcal{H}})=\sum_{P \in \tilde{\mathcal{S}}}\left(\operatorname{mult}_{P}(\tilde{\Phi}, \mathcal{C})-\operatorname{mult}_{P}(\Phi, \mathcal{C})\right)
$$

where mult ${ }_{P}\left(\mathcal{C}_{1}, \mathcal{C}_{2}\right)$ denotes the multiplicity of intersection of the curves $\mathcal{C}_{1}, \mathcal{C}_{2}$ at the point $P$.

Essentially, this notion requires that when a point or a family of points on $\mathcal{C}$ are used to generate a subsystem $\tilde{\mathcal{H}}$ of $\mathcal{H}$ (by introducing some points on $\mathcal{C}$ as new base point on $\mathcal{H}$ with specific multiplicities) the linear system of equations containing the contraints is over $\mathbb{L}$, and its rank equals the number of new known intersection points between $\mathcal{C}$ and a generic representative of the subsystem. In the next proposition some special cases of rational linear subsystem are analyzed. The following Proposition 1 and Theorem 2 are proved in [SW97].

Proposition 1: Let $\mathcal{C}$ be a rational plane curve of degree $d$ defined by a polynomial over $\mathbb{L}, \mathcal{H}_{a}$ the linear system of adjoint curves to $\mathcal{C}$ of degree $a \in$ $\{d, d-1, d-2\}$, and $\mathcal{F}=\left\{\left(p_{1}(t): p_{2}(t): p_{3}(t)\right)\right\}_{A(t)}$ a family of $k$ conjugate points on $\mathcal{C}$ over $\mathbb{L}$. Then we have the following:

(i) If $\mathcal{F}$ is a family of simple points, $k \leq \operatorname{dim}\left(\mathcal{H}_{a}\right)$, and $\tilde{\mathcal{H}}_{a}$ is the subsystem of $\mathcal{H}_{a}$ obtained by forcing every point in $\mathcal{F}$ to be a simple base point of $\tilde{\mathcal{H}}_{a}$, then $\tilde{\mathcal{H}}_{a}$ is rational, and $\operatorname{dim}\left(\tilde{\mathcal{H}}_{a}\right)=\operatorname{dim}\left(\mathcal{H}_{a}\right)-k$.

(ii) If $\mathcal{F}$ is a family of $r$-fold points, $r \cdot k \leq \operatorname{dim}\left(\mathcal{H}_{a}\right)$, and $\tilde{\mathcal{H}}_{a}$ is the subsystem of $\mathcal{H}_{a}$ obtained by forcing every point in $\mathcal{F}$ to be a base point of $\tilde{\mathcal{H}}_{a}$ of multiplicity $r$, then $\tilde{\mathcal{H}}_{a}$ is rational, and $\operatorname{dim}\left(\tilde{\mathcal{H}}_{a}\right)=\operatorname{dim}\left(\mathcal{H}_{a}\right)-r k$.

Theorem 2: Let $\mathcal{C}$ be a rational plane curve of degree $d$ defined by a polynomial over $\mathbb{L}$, and $\mathcal{H}_{a}$ the linear system of adjoint curves to $\mathcal{C}$ of degree $a \in\{d, d-$ $1, d-2\}$. Then every rational linear subsystem of $\mathcal{H}_{a}$ of dimension $s$ with all its base points on $\mathcal{C}$ provides curves that generate families of $s$ conjugate simple points over $\mathbb{L}$ by intersection with $\mathcal{C}$.

As a consequence of Proposition 1 and Theorem 2 we get the following algorithmically important facts.

Theorem 3: Let $\mathcal{C}$ be a rational plane curve of degree $d$, defined by a polynomial $f(x, y) \in \mathbb{L}[x, y]$. 
(i) $\mathcal{C}$ has families of $d-2,2 d-2$, and $3 d-2$ conjugate simple points over $\mathbb{L}$.

(ii) $\mathcal{C}$ has families of 2 conjugate simple points over $\mathbb{L}$.

(iii) If $d$ is odd, then $\mathcal{C}$ has a simple point over $\mathbb{L}$.

(iv) If $d$ is even, then $\mathcal{C}$ has simple points over an algebraic extension of $\mathbb{L}$ of degree 2.

Proof: (i) Let $P_{1}, \ldots, P_{n}$ be the singular points on $\mathcal{C}$, having multiplicities $r_{1}, \ldots, r_{n}$, respectively. Since we assume that all singularities are ordinary and $\mathcal{C}$ is rational, we have

$$
(d-1)(d-2)=\sum_{i=1}^{n} r_{i}\left(r_{i}-1\right)
$$

By application of Proposition 1 we see that the dimension of the system of adjoint curves of degree $d-2, \mathcal{H}_{d-2}$, to $\mathcal{C}$ is

$$
\frac{(d-1) d}{2}-1-\sum_{i=1}^{n} \frac{\left(r_{i}-1\right) r_{i}}{2}=d-2 .
$$

Now we can apply Theorem 2 for $s=d-2$ (i.e. choosing the whole system) and we get that $\mathcal{C}$ has families of $d-2$ conjugate simple points. Similarly, by using systems of adjoint curves of degrees $d-1$ and $d$, respectively, we get that $\mathcal{C}$ has families of $2 d-2$ and $3 d-2$ conjugate simple points.

(ii) We first apply statement (i) to obtain two different families of $(d-2)$ simple points. Let $\mathcal{H}_{d-1}$ be the system of adjoint curves of degree $(d-1)$. Applying Proposition 1 one has that the linear subsystem $\tilde{\mathcal{H}}_{d-1}$ obtained by forcing all the points in these two families to be simple base points of $\mathcal{H}_{d-1}$ is rational of dimension 2. Thus, applying Theorem 2 to $\tilde{\mathcal{H}}_{d-1}$ one obtains families of two simple points.

(iii) Applying statement (ii) one can determine $\frac{d-3}{2}$ different families of two simple points on $\mathcal{C}$. Let $\mathcal{H}_{d-2}$ be the system of adjoint curves of degree $(d-2)$. Applying Proposition 1 one has that the linear subsystem $\tilde{\mathcal{H}}_{d-2}$ obtained by forcing all the points in these families to be simple base points of $\mathcal{H}_{d-2}$ is rational of dimension one. Thus, applying Theorem 2 one concludes that $\mathcal{C}$ has simple points over $\mathbb{L}$.

(iv) This is an inmediate consequence of statement (ii).

Summarizing we get the following algorithm for deciding the parametrizability over $\mathbb{R}$ and, in the positive case, computing such a parametrization.

\section{Algorithm REAL-PARAM $(f)$}

- Input: $F\left(x_{1}, x_{2}, x_{3}\right) \in \mathbb{L}\left[x_{1}, x_{2}, x_{3}\right]$ is an irreducible homogeneous polynomial of degree $d$, that defines a rational plane curve $\mathcal{C}$.

- Output: a real parametrization of $\mathcal{C}$, or "no-real-parametrization" if no real parametrization exists.

(1) Compute the linear system $H$ of adjoint curves to $\mathcal{C}$ of degree $(d-2)$. 
(2) If $d$ is odd, apply Theorem 3 (iii) to find $(d-3)$ simple points of $F$ over $\mathbb{L}$.

(3) If $d$ is even, apply Theorem 3 (ii) to find $\frac{d-4}{2}$ families of two simple points of $F$ over $\mathbb{L}$.

(4) Determine the linear rational subsystem $\tilde{H}$ obtained by forcing the points computed in steps (2) and (3) to be simple base points on $H$.

(5) Take $\tilde{\Phi}_{1}, \tilde{\Phi}_{2}, \tilde{\Phi}_{3} \in \tilde{H}$ such that the common intersections of the three curves $\tilde{\Phi}_{i}$ and $F$ are the set of base points of $\tilde{H}$, and such that

$$
\mathcal{T}=\left\{y_{1}: y_{2}: y_{3}=\tilde{\Phi}_{1}: \tilde{\Phi}_{2}: \tilde{\Phi}_{3}\right\}
$$

is a birational transformation (Theorem 1).

(6) Determine the transformed curve $\mathcal{D}$ to $\mathcal{C}$ obtained by $\mathcal{T}$. Note that applying Theorem 1 one has that $\mathcal{D}$ is either a conic or a line depending on whether $d$ is even or odd, respectively. $\mathcal{D}$ can be easily determined by sending a few points from $\mathcal{C}$ to $\mathcal{D}$ and then interpolating.

(7) If $d$ is odd, parametrize the line $\mathcal{D}$ over $\mathbb{L}$. Apply the inverse transformation $\mathcal{T}^{-1}$ to find a parametrization of $\mathcal{C}$ over $\mathbb{L}$, and therefore over $\mathbb{R}$. (Or, alternatively, determine as many points on $\mathcal{D}$ over $\mathbb{L}$ as necessary, transfer them back to $\mathcal{C}$ by $\mathcal{T}^{-1}$, and use them for computing a parametrization of $\mathcal{C}$ over L.)

(8) If $d$ is even, decide whether the conic $\mathcal{D}$ can be parametrized over $\mathbb{R}$. If so, parametrize $\mathcal{D}$ over $\mathbb{R}$. Apply the inverse transformation $\mathcal{T}^{-1}$ to find a real parametrization of $\mathcal{C}$ over $\mathbb{R}$. (Or, alternatively, determine as many points on $\mathcal{D}$ over $\mathbb{R}$ as necessary, transfer them back to $\mathcal{C}$ by $\mathcal{T}^{-1}$, and use them for computing a parametrization of $\mathcal{C}$ over $\mathbb{R}$.)

If not, report "no-real-parametrization".

In step (8) we have to decide whether an irreducible conic $\mathcal{D}$, defined by a homogeneous polynomial $G\left(y_{1}, y_{2}, y_{3}\right) \in \mathbb{L}\left[y_{1}, y_{2}, y_{3}\right]$, contains a real point $P$. If so, then we can obviously parametrize $\mathcal{D}$ by intersecting it by lines through $P$.

In fact we can decide whether $\mathcal{D}$ contains a rational point. For details see [?] and [?]. If this is not the case, we can transform $\mathcal{D}$ to an equivalent conic $\mathcal{D}^{\prime}$ by a birational mapping over $\mathbb{R}$, such that the defining equation of $\mathcal{D}^{\prime}$ has the form

$$
y_{1}^{2} \pm y_{2}^{2} \pm y_{3}^{2}=0
$$

(see, e.g., the Law of Inertia in [?]). Then $\mathcal{D}^{\prime}$, and hence $\mathcal{D}$, has a real point if and only if the defining equation of $\mathcal{D}^{\prime}$ is not equivalent to $y_{1}^{2}+y_{2}^{2}+y_{3}^{2}=0$. In fact, if $\mathcal{D}$ contains a real point, then we can compute one. Thus, the question in step (8) can be completely decided.

An alternative approach is to decide the reality of $\mathcal{C}$ by computing the signature of the corresponding quadratic form, and once the reality is decided, to find a family of two conjugate points on $\mathcal{C}$ (Theorem 3 (ii)) whose quadratic defining polynomial has real roots. 


\section{Real reparametrization}

If a complex rational parametization $\mathcal{P}(t)$ of an irreducible affine plane curve $\mathcal{C}$ over $\mathbb{C}$ is given, or computed by any parametrization algorithm, the alternative approach presented in [?] may be considered. In this situation, the reality of $\mathcal{C}$ is decided by computing a gcd of two real bivariate polynomonials, and if the curve is real, a linear parameter change is determined to transform the original parametrization into a real one.

The main idea of the algorithm presented in [?] is to associate with the original parametrization a plane curve that contains as points the complex values (taking the real and imaginary parts) of the parameter that generates, via the parametrization, the real points on the original curve. Then the reality of the original curve is characterized by means of the reality of the associated curve, that is proved to be either a line or a circle. More precisely, let $\mathcal{P}(t)$ be the proper complex parametrization of $\mathcal{C}$ :

$$
x(t)=\frac{q_{1}(t)}{h(t)}, \quad y(t)=\frac{q_{2}(t)}{h(t)},
$$

where $q_{1}, q_{2}, h \in \mathbb{C}[t]$ and $\operatorname{gcd}\left(q_{1}, q_{2}, h\right)=1$. Then, we apply the formal change of variable $t=t_{1}+i t_{2}$ to $\mathcal{P}(t)$ to obtain:

$$
\begin{aligned}
& x\left(t_{1}+i t_{2}\right)=\frac{u_{1}\left(t_{1}, t_{2}\right)+i v_{1}\left(t_{1}, t_{2}\right)}{h_{1}\left(t_{1}, t_{2}\right)^{2}+h_{2}\left(t_{1}, t_{2}\right)^{2}}, \\
& y\left(t_{1}+i t_{2}\right)=\frac{u_{2}\left(t_{1}, t_{2}\right)+i v_{2}\left(t_{1}, t_{2}\right)}{h_{1}\left(t_{1}, t_{2}\right)^{2}+h_{2}\left(t_{1}, t_{2}\right)^{2}}
\end{aligned}
$$

where $h_{1}, h_{2} \in \mathbb{R}\left[t_{1}, t_{2}\right], u_{1}, v_{1} \in \mathbb{R}\left[t_{1}, t_{2}\right]$ and $u_{2}, v_{2} \in \mathbb{R}\left[t_{1}, t_{2}\right]$ are the real and imaginary parts of $h\left(t_{1}+i t_{2}\right), q_{1}\left(t_{1}+i t_{2}\right) \cdot \bar{h}\left(t_{1}-i t_{2}\right)$ and $q_{2}\left(t_{1}+i t_{2}\right) \cdot \bar{h}\left(t_{1}-i t_{2}\right)$, respectively $(\bar{h}$ denotes here the conjugate of $h)$. Then, it is proved in [?] that the plane curve $\mathcal{C}$ is real if and only if $\operatorname{gcd}\left(v_{1}, v_{2}\right)$ is either a real line or a real circle. Furthermore, if the plane curve $\mathcal{C}$ is real, and $\left(m_{1}(t), m_{2}(t)\right)$ is a real proper rational parametrization of $\operatorname{gcd}\left(v_{1}, v_{2}\right)$, then $\mathcal{P}\left(m_{1}(t)+i m_{2}(t)\right)$ is a real proper rational parametrization of $\mathcal{C}$.

Clearly, these two results provide an algorithm for deciding the reality of curves, and in the affirmative case, computing the linear change of parameter that reparametrizes the original complex proper parametrization into a real proper parametrization.

\section{Examples}

Example 2: We consider the curve $\mathcal{C}_{1}$ of Example 1. $\mathcal{C}_{1}$ is defined by

$$
f_{1}(x, y)=\left(x^{2}+4 y+y^{2}\right)^{2}-16\left(x^{2}+y^{2}\right)=0 .
$$

Let us first apply the algorithm REAL-PARAM to $\mathcal{C}_{1}$ to see whether it is parametrizable. 
$\mathcal{C}_{1}$ has 3 double points in the projective plane, namely

$$
(0: 0: 1) \text { and }(1: \pm i: 0) \text {. }
$$

So genus $\left(\mathcal{C}_{1}\right)=0$, which means that $\mathcal{C}_{1}$ is rational and must have a parametrization over $\mathbb{C}$ (the picture actually suggests that it is a real curve, and therefore must have a parametrization over $\mathbb{R}$ ).

The system $\tilde{\mathcal{H}}$ of conics (curve of degree 2) passing through all three of these double points is defined by

$$
h(x, y, z, s, t)=x^{2}+s x z+y^{2}+t y z,
$$

so it is a system of dimension 2. Let the birational transformation $\mathcal{T}$ be

$$
\mathcal{T}=\left(\Phi_{1}: \Phi_{2}: \Phi_{3}\right)=(h(x, y, z, 0,1): h(x, y, z, 1,0): h(x, y, z, 1,1)),
$$

i.e.

$$
\Phi_{1}=x^{2}+y^{2}+y z, \quad \Phi_{2}=x^{2}+x z+y^{2}, \quad \Phi_{3}=x^{2}+x z+y^{2}+y z .
$$

We determine the birationally equivalent conic $\mathcal{D}_{1}$ to $\mathcal{C}_{1}$ by sending the 6 points in the families

$$
\begin{aligned}
& \mathcal{F}_{1}=\left\{(t:-t+2: 1) \mid 4 t^{4}-32 t^{3}+80 t^{2}-128 t+80\right\}, \\
& \mathcal{F}_{2}=\left\{(t: 1-2 t: 1) \mid t^{2}-4 t+1\right\}
\end{aligned}
$$

onto $\mathcal{D}_{1}$ by $\mathcal{T}$. This gives us the conic defined by

$$
15 x^{2}+7 y^{2}+6 x y-38 x-14 y+23
$$

$\mathcal{D}_{1}$ has the real (in fact, rational) point $(1,8 / 7)$, which (by $\mathcal{T}^{-1}$ ) corresponds to the point $P=(0,-8)$ on $\mathcal{C}_{1}$.

Now we restrict $\tilde{\mathcal{H}}$ to the curves through $P$. This restricted linear system is defined by (after renaming of the free parameter)

$$
h^{\star}(x, y, z, t)=x^{2}+t x z+y^{2}+8 y z .
$$

Computing the resultants of $f_{1}(x, y)$ and $h^{\star}(x, y, 1, t)$, with respect to $x$ and $y$, respectively, and taking the primitive parts with respect to the parameter $t$, one gets two polynomials $R_{1} \in \mathbb{C}[y, t]$ and $R_{2} \in \mathbb{C}[x, t]$, such that the degrees of $R_{1}$ and $R_{2}$, with respect to $y$ and $x$, respectively, are one. Hence, solving the system $\left\{R_{1}=0, R_{2}=0\right\}$ in the variables $\{x, y\}$, one gets the following real parametrization $\mathcal{P}(t)$ of $\mathcal{C}_{1}$ :

$$
x(t)=\frac{-1024 t^{3}}{256 t^{4}+32 t^{2}+1}, \quad y(t)=\frac{-2048 t^{4}+128 t^{2}}{256 t^{4}+32 t^{2}+1} .
$$

Let us now apply the real reparametrization approach of section 4 to $\mathcal{C}_{1}$. The idea is, therefore, to apply any basic parametrization algorithm to $\mathcal{C}_{1}$ without taking care of the field extensions, and afterwards to analyze the possible reparametrization of the achieved parametrization over the ground field. Thus, 
the first steps are the same. We consider the linear system $\tilde{\mathcal{H}}$ of adjoint curves of degree 2 , and then we force $\tilde{\mathcal{H}}$ to pass through any simple point on the curve. We take, for instance, $Q=\left(-\frac{128}{9} i:-\frac{160}{9}: 1\right)$. The obtained linear subsystem of $\tilde{\mathcal{H}}$ has dimension 1 and is defined by the polynomial

$$
h^{\star}(x, y, z, t)=x^{2}+t x z+y^{2}+\left(\frac{34}{5}-\frac{4}{5} i t\right) y z .
$$

Now, proceeding as above, we get the following parametrization $\mathcal{Q}(t)$ over $\mathbb{C}$ of $\mathcal{C}_{1}$ :

$$
\begin{aligned}
& x(t)=-32 \frac{-1024 i+128 t-144 i t^{2}+i t^{4}-22 t^{3}}{2304-3072 i t-736 t^{2}+9 t^{4}-192 i t^{3}} \\
& y(t)=-40 \frac{1024-256 i t-80 t^{2}+t^{4}+16 i t^{3}}{2304-3072 i t-736 t^{2}+9 t^{4}-192 i t^{3}} .
\end{aligned}
$$

Now, we execute the formal change of parameter $t=t_{1}+i t_{2}$ in $\mathcal{Q}(t)$, and we compute the gcd of the imaginary parts, $v_{1}\left(t_{1}, t_{2}\right)$ and $v_{2}\left(t_{1}, t_{2}\right)$, of the normalized (i.e. with denominators in $\left.\mathbb{R}\left[t_{1}, t_{2}\right]\right)$ rational functions $x\left(t_{1}+i t_{2}\right)$ and $y\left(t_{1}+i t_{2}\right)$, respectively:

$$
D\left(t_{1}, t_{2}\right)=\operatorname{gcd}\left(v_{1}, v_{2}\right)=t_{1}^{2}+t_{2}^{2}+6 t_{2}-16 .
$$

In this situation, since $D\left(t_{1}, t_{2}\right)$ defines a real circle, it follows that the original curve $\mathcal{C}_{1}$ is real and, therefore, parametrizing over the reals the curve defined by $D\left(t_{1}, t_{2}\right)$ one gets the linear change of parameter to transform $\mathcal{Q}(t)$ into a real parametrization. More precisely, one takes the real parametrization of $D\left(t_{1}, t_{2}\right)$ :

$$
t_{1}(t)=\frac{-10 t}{t^{2}+1}, \quad t_{2}(t)=\frac{-10 t}{t^{2}+1} .
$$

Therefore, $\mathcal{L}(t)=\mathcal{Q}\left(\frac{-10 t}{t^{2}+1}+i \frac{-10 t}{t^{2}+1}\right)$ is a real parametrization of $\mathcal{C}_{1}$. In fact, $\mathcal{L}(t)$ is the parametrization:

$$
x(t)=\frac{-32 t}{16 t^{4}+8 t^{2}+1}, \quad y(t)=8 \frac{4 t^{4}-1}{16 t^{4}+8 t^{2}+1} .
$$

Example 3: We consider the curve $\mathcal{C}_{2}$ of Example 1. $\mathcal{C}_{2}$ is defined by

$$
f_{2}(x, y)=2 y^{2}+x^{2}+2 x^{2} y^{2}=0 .
$$

Let us apply the algorithm REAL-PARAM to $\mathcal{C}_{2}$ to see whether it can be parametrized. The singularitities of $\mathcal{C}_{2}$ in the projective plane are

$$
(1: 0: 0), \quad(0: 1: 0), \quad(0: 0: 1),
$$

each of which is a double point. So genus $\left(\mathcal{C}_{2}\right)=0$, which means that $\mathcal{C}_{2}$ can be parametrized over $\mathbb{C}$. 
The system $\tilde{\mathcal{H}}$ of conics passing through all three of these double points is defined by

$$
h(x, y, z, s, t)=x z+t y z+s x y,
$$

so it is a system of dimension 2. Let the birational transformation $\mathcal{T}$ be

$$
\mathcal{T}=\left(\Phi_{1}: \Phi_{2}: \Phi_{3}\right)=(h(x, y, z, 1,0): h(x, y, z, 0,1): h(x, y, z, 1,1)),
$$

i.e.

$$
\Phi_{1}=x z+x y, \quad \Phi_{2}=x z+y z, \quad \Phi_{3}=x z+y z+x y .
$$

We determine the birationally equivalent conic $\mathcal{D}_{2}$ to $\mathcal{C}_{2}$ by sending the 8 points in the families

$$
\begin{aligned}
& \mathcal{F}_{1}=\left\{(t:-2 t+1: 1) \mid 8 t^{4}-8 t^{3}+11 t^{2}-8 t+2\right\} \\
& \mathcal{F}_{2}=\left\{(t:-t+2: 1) \mid 2 t^{4}-8 t^{3}+11 t^{2}-8 t+8\right\}
\end{aligned}
$$

onto $\mathcal{D}_{2}$ by $\mathcal{T}$. This gives us the conic defined by

$$
5 z^{2}-6 x z-6 y z+3 x^{2}+2 x y+3 y^{2} .
$$

$\mathcal{D}_{2}$ has no real point. So also $\mathcal{C}_{2}$ can have no real point, i.e. it is NOT parametrizable over $\mathbb{R}$.

But we can parametrize $\mathcal{C}_{2}$ over $\mathbb{C}$ by passing the system of adjoint curves through the point

$$
P=(-\alpha, \alpha), \quad \text { where } 2 \alpha^{2}+3=0,
$$

getting

$$
h^{\star}(x, y, z, t)=x z+t y z+\frac{2}{3}(\alpha-\alpha t) x y .
$$

Now, proceeding as in Example 2, we get the following parametrization $\mathcal{P}(t)$ of $\mathcal{C}_{2}$ over $\mathbb{C}$ :

$$
x(t)=\frac{-\alpha t^{2}-2 \alpha}{t^{2}-2 t-2}, \quad y(t)=\frac{-\alpha t^{2}-2 \alpha}{t^{2}+4 t-2} .
$$

We leave the application of the reparametrization algorithm to the reader.

\section{Conclusion}

So, as we have seen above, we can parametrize any parametrizable real curve by a real parametrization $\mathcal{P}(t)=(x(t), y(t))$, i.e. $x(t), y(t) \in \mathbb{R}(t)$. This is what we usually need in applications, such as in computer aided geometric design. The algorithms described in this paper allow us, for the first time, to decide the possibility of a real parametrization and, if it exists, to actually compute one. 


\section{References}

[AB88] Abhyankar S.S, Bajaj C.L., (1988), Automatic parametrization of rational curves and surfaces III: Algebraic plane curves. Computer Aided Geometric Design 5, 309-321.

[Ga59] Gantmacher F.R., (1959), The Theory of Matrices. Chelsea, New York.

[HH90] Hilbert D., Hurwitz A. (1890), Über die Diophantischen Gleichungen vom Geschlecht Null. Acta math. 14, 217-224.

[HW98] Hillgarter E., Winkler F. (1998), Points on algebraic curves and the parametrization problem. In: D. Wang (ed.), Automated Deduction in Geometry, 185-203, Lecture Notes in Artif. Intell. 1360, Springer Verlag Berlin Heidelberg.

[vH97] van Hoeij M. (1997), Rational parametrizations of algebraic curves using a canonical divisor. J. Symbolic Computation 23/2\&3, 209-227.

[IR82] Ireland K., Rosen R. (1982), A classical introduction to modern number theory. Springer Verlag, Graduate Texts in Mathematics, New York.

[MSW96] Mňuk M., Sendra J.R., Winkler F. (1996), On the complexity of parametrizing curves. Beiträge zur Algebra und Geometrie 37/2, 309-328.

[MW96] Mňuk M., Winkler F. (1996), CASA - A System for Computer Aided Constructive Algebraic Geometry. In: J. Calmet and C. Limongelli (eds.), Proc. Internat. Symp. on Design and Implementation of Symbolic Computation Systems (DISCO'96), 297-307, LNCS 1128, Springer Verlag Berlin Heidelberg New York.

[RS95] Recio T., Sendra J.R. (1995), Reparametrización real de curvas reales paramétricas. Proc. EACA'95, 159-168, Univ. de Cantabria, Santander, Spain.

[RS97a] Recio T., Sendra J.R. (1997), Real parametrizations of real curves. J. Symbolic Computation 23/2\&3, 241-254.

[RS97b] Recio T., Sendra J.R. (1997), A really elementary proof of real Lüroth's theorem. Revista Matemática de la Universidad Complutense de Madrid 10, 283291.

[SW91] Sendra J.R., Winkler F. (1991), Symbolic parametrization of curves. J. Symbolic Computation 12/6, 607-631.

[SW97] Sendra J.R., Winkler F. (1997), Parametrization of algebraic curves over optimal field extensions. J. Symbolic Computation 23/2\&3, 191-207.

[Wa50] Walker R.J. (1950), Algebraic curves. Princeton Unversity Press.

[Wi96] Winkler F. (1996), Polynomial algorithms in computer algebra. Springer-Verlag Wien New York.

This article was processed using the $\mathrm{IAT}_{\mathrm{E}} \mathrm{X}$ macro package with LLNCS style 